\title{
Transport and divertor properties of the dynamic ergodic divertor
}

\author{
M Lehnen $^{1,5}$, S Abdullaev ${ }^{1}$, W Biel ${ }^{1}$, M F M de Bock ${ }^{2}$, S Brezinsek $^{1}$,

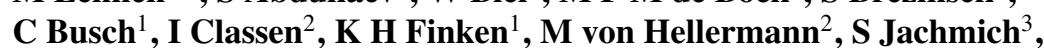 \\ M Jakubowski ${ }^{1}$, R Jaspers ${ }^{2}$, H R Koslowski ${ }^{1}$, A Krämer-Flecken ${ }^{1}$, \\ Y Kikuchi ${ }^{1}$, Y Liang ${ }^{1}$, A Nicolai ${ }^{1}$, A Pospieszczyk ${ }^{1}$, T Van Rompuy ${ }^{4}$, \\ U Samm $^{1}$, O Schmitz ${ }^{1}$, G Sergienko ${ }^{1}$, B Unterberg ${ }^{1}$, R Wolf ${ }^{1}$, \\ O Zimmermann ${ }^{1}$ and the TEXTOR team

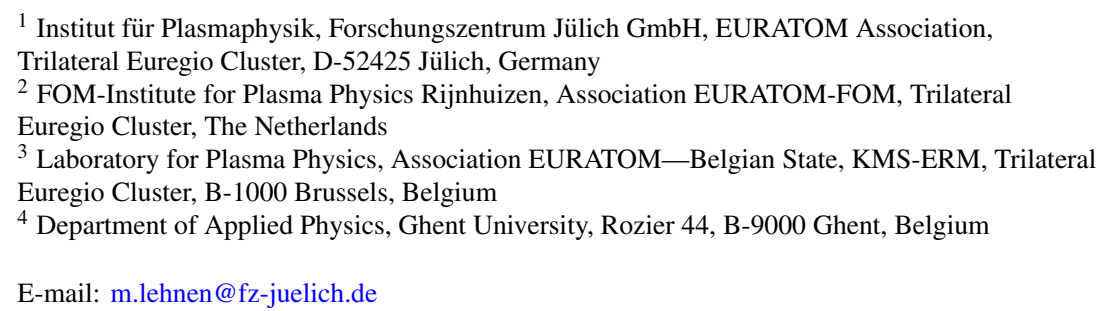

Received 12 October 2005

Published

Online at stacks.iop.org/PPCF/47

\begin{abstract}
The concept of the dynamic ergodic divertor (DED) is based on plasma edge ergodization by a resonant perturbation. Such a divertor concept is closely related to helical or island divertors in stellerators. The base mode of the DED perturbation field can be $m / n=12 / 4,6 / 2$ or $3 / 1$. The $3 / 1$ base mode with its deep penetration of the perturbation field provides the excitation of tearing modes. This topic was presented elsewhere. In this contribution we concentrate on the divertor properties of the DED. We report on the characterization of the topology, transport properties in ergodic fields, impurity transport and density limit behaviour.

The $12 / 4$ base where the perturbation is restricted to the plasma edge is suitable for divertor operation. With increasing perturbation field island chains are built up at the resonance layers. Overlapping islands lead to ergodization. The plasma is guided in the laminar region via open field lines of short connection length to the divertor target. The magnetic topology is not only controlled by the coil current but especially by the edge safety factor. For appropriate edge safety factor we observe a strong temperature drop in the plasma edge, indicating an expansion of the laminar region, which is necessary to decouple the divertor plasma from the core plasma. The modifications of
\end{abstract}

\footnotetext{
5 Author to whom any correspondence should be addressed.
} 
the magnetic topology can be directly seen, for example, from carbon emission lines. The magnetic structure is calculated by the ATLAS code and shows good agreement with the experimental findings.

(Some figures in this article are in colour only in the electronic version)

\section{Introduction}

Stochastic field structures are applied in fusion devices for various purposes. The first application was to control the plasma edge transport in an ergodic divertor. As in the standard divertor the aim is the safe and efficient particle and heat exhaust together with a reduced impurity contamination of the plasma. Examples for this application include not only the ergodic divertor at Tore Supra [1] but also island divertor concepts like in LHD [2] or as planned in W7-X [3]. Beside the divertor programme, perturbation fields are applied for influencing MHD activities. The control of large ELM activities in fusion relevant H-mode plasmas is an urgent task and the application of perturbation fields has shown to be a very promising technique to suppress ELMs [4]. The dynamic ergodic divertor (DED) is, with its possibility to create rotating perturbations, a natural continuation of the divertor programme. It is also a tool to control MHD activities and plasma rotation which gives access to various studies like, for example, the suppression of tearing modes or the influence of error fields on plasma stability. These topics are beyond the scope of this paper, and the reader is referred to [5-8].

The DED creates a resonant perturbation at the plasma edge. The spectrum of the perturbation field is controlled by the current distribution in the 16 divertor coils at the high field side of TEXTOR [9]. The coils cover about $72^{\circ}$ of the minor circumference resulting in a broader mode spectrum. Two operating modes were investigated with base mode numbers $m / n=3 / 1$ and $m / n=12 / 4$. The current direction in the coils is alternating with two (eight for $m / n=3 / 1$ ) adjacent coils having the same current. These two (eight) coils are fed by two power supplies which run in AC operation with a $\pm 90^{\circ}$ phase shift to produce a rotating perturbation field. The rotation direction is defined as 'co-rotation' for a field moving in the direction of the plasma current. The maximum current per coil is $15 \mathrm{kA}(3.75 \mathrm{kA}$ for $m / n=3 / 1$ ). The two operation scenarios differ in the penetration depth of the perturbation field. The perturbed volume for $m / n=12 / 4$ is restricted to about $10 \%$ of the plasma minor radius. The deeply penetrating perturbation field in the $3 / 1$ base mode is likely to excite tearing modes with mode numbers $m / n=2 / 1$ and at higher perturbation level $m / n=3 / 1$. Thus the $12 / 4$ configuration was naturally chosen for divertor studies. It was recently found, however, that the threshold for mode onset can be significantly increased by NBI counter injection. In discharges with pure counter injection, the maximum perturbation field could be applied without the excitation of a tearing mode. This makes the $3 / 1$ base mode attractive for the analysis of the divertor operation as well, although the choice of scenario is restricted.

First descriptions of the divertor properties of the DED can be found in [10,11]. This paper gives an overview of the recently achieved results in divertor physics with focus on topology and transport. The basis for the transport analysis is the knowledge about the three-dimensional divertor topology, which is introduced in section 2. The influence of the edge safety factor on the transport properties in the divertor volume and towards the target plates is discussed in section 3. The dependence of the plasma rotation on the perturbation is shown in section 4 . An simple estimate for the electric field and the toroidal rotation is given. The particle recycling in the ergodic divertor and its influence on the density limit and impurity transport is analysed in section 5 . 

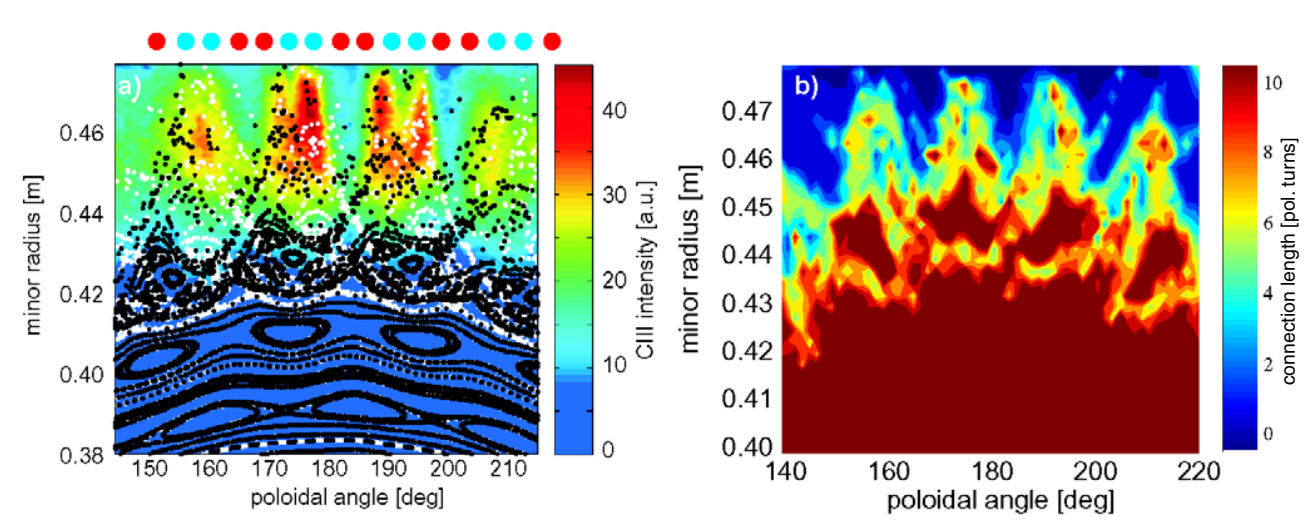

Figure 1. 12/4 configuration: $\mathrm{C}_{\text {III }}$ emission in front of the target plate overlayed with a Poincaré plot for discharge \#96018. Black and white dots indicate the toroidal direction of field line tracing (white/black: co/counter-current direction). The DED current distribution is given by the red and blue dots. (b) Connection length in the laminar region.

\section{Divertor topology}

The divertor volume splits into two regions, the ergodic region adjacent to the confined plasma region and the laminar region close to the target plates with field lines of short connection length to the target. A geometrical argument defines the ergodic region as a region with field lines of connection length $L_{\mathrm{c}}$ much larger than the Kolmogorov length $L_{\mathrm{k}}$. Field line tracing using a mapping technique [12] shows that the width of the ergodic region is small in the $12 / 4$ configuration and also inhomogeneous along the poloidal circumference. Field line diffusion coefficients are below $3 \times 10^{-6} \mathrm{~m}^{6}$. In the $3 / 1$ configuration a much broader ergodic region is predicted which extends up to the $q=2$ surface at higher perturbation amplitude. The field line diffusion can reach values up to $D_{\mathrm{FL}}=5 \times 10^{-5} \mathrm{~m}$.

Figure 1(a) shows the $\mathrm{C}$ III radiation in front of the divertor target at the high field side (HFS). The emission depends on the carbon density but also reflects the variation of the plasma parameters. The discharge parameters are a plasma current $I_{\mathrm{P}}=320 \mathrm{kA}$ and a toroidal field $B_{\mathrm{t}}=1.9 \mathrm{~T}$ which result in an edge safety factor $q_{\mathrm{a}}=3.1$. Moderate neutral beam heating with total power $P_{\mathrm{NBI}}=500 \mathrm{~kW}$ was applied. The coil current in the DED is $I_{\mathrm{DED}}=11.5 \mathrm{kA}$, which is $75 \%$ of the maximum. The strongest $\mathrm{C}$ III emission is seen in front of the eight strike lines at the target. The position of these strike lines is defined by a finger like structure. Within this structure we find field lines with $L_{\mathrm{c}} \gg 1$ p.t. (p.t. = poloidal turns) connecting the ergodic region to the target plates. These field lines stay for many poloidal turns inside the ergodic region and are then within one or two poloidal turns deflected towards the target. The magnetic structure is given by the Poincaré plot overlayed. The dots are coloured black and white to indicate the different toroidal direction of the field line tracing. The structure of the laminar region is given by the contour plot of the field line connection length in figure $1(b)$. The laminar volume with $L_{\mathrm{c}} \leqslant 2$ p.t. is quite small and riddled with areas of field lines with long connection length.

The topology of the $3 / 1$ configuration differs significantly from that of the $12 / 4$, as can be seen from figure 2 . The discharge shown is predominantly heated with counter NBI

6 This value was calculated from field line mapping taking into account the inhomogenous structure of the ergodic region [12]. This procedure usually gives lower values compared to the quasilinear theory [15] since it takes into account the laminar behaviour of the field lines close to the perturbation coils. 

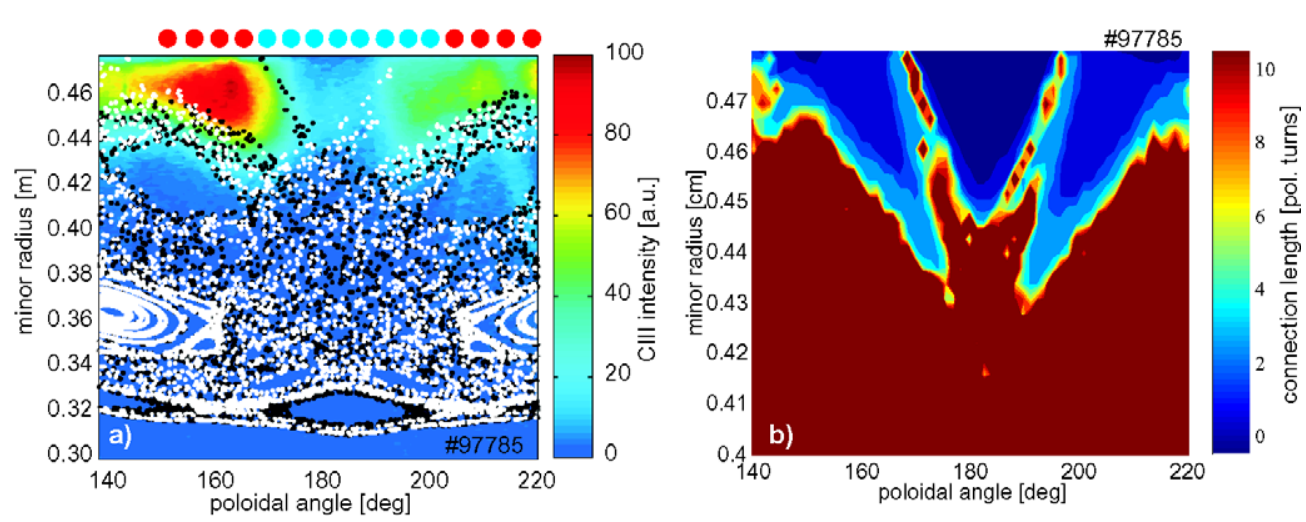

Figure 2. 3/1 configuration: (a) $\mathrm{C}$ III emission in front of the target plate overlayed with a Poincaré plot for discharge \#97785. Black and white dots indicate the toroidal direction of field line tracing (white/black: co/counter-current direction). The DED current distribution is given by the red and blue dots. (b) Connection length in the laminar region.

$\left(P_{\mathrm{NBI}}=800 \mathrm{~kW}\right)$ in order to increase the threshold for exciting a $m / n=2 / 1$ tearing mode. The example given is for $I_{\mathrm{DED}}=2.0 \mathrm{kA}$ ( $53 \%$ of the maximum amplitude) which is just below the threshold for the tearing mode. The safety factor is chosen to be $q_{\mathrm{a}}=5$ to reduce the perturbation strength at the $q=2$ surface $\left(I_{\mathrm{P}}=300 \mathrm{kA}\right.$ and $\left.B_{\mathrm{t}}=2.25 \mathrm{~T}\right)$. Only two strike lines form on the divertor target. The finger structure is very thin and the laminar volume is large. The area in between the fingers is the private flux region with $L_{\mathrm{c}}<1$ whereas the region on the left and right of the two fingers has the properties of a scrape-off layer (SOL) as in a poloidal divertor. The strong difference of the radiation in the upper and lower strike point might indicate a flow asymmetry as observed in poloidal divertors. This effect needs further investigations, in particular in discharges with reversed plasma current and toroidal field. However, some care is needed in the interpretation of these signals since the radiation intensity also depends on the vertical plasma position and the alignment of the target tiles.

Figure 3 shows an overview of the structure for the whole poloidal circumference. It can be seen that ergodic regions $\left(L_{\mathrm{k}} \ll L_{\mathrm{c}}\right)$ alternate with laminar flux tubes $\left(L_{\mathrm{k}} \approx L_{\mathrm{c}}\right)$. The laminar flux tubes connect within one poloidal turn to the target as indicated by the red dots. Inside these flux tubes the parallel transport to the target dominates resulting in a particle and heat sink at these positions. Measurements at the LFS with the ECE imaging diagnostic (right-hand side of figure 3) and the helium beam diagnostic [13] show a strong drop in the electron temperature inside these flux tubes (magenta box). Inside the ergodic regions (blue box) an enhanced radial transport leads to a flattening of the temperature profiles. However, it is important to note that the ergodic regions are poloidally localized with poloidal mode number $m=11$ in this case. Accordingly, the electron pressure can vary by up to a factor of 2 in the poloidal direction [13]. A strong radial modulation as found at Tore Supra [1] is not observed. This might be caused by the lower degree of ergodization in the DED case where only one rational $q$-surface (in this example $q=11 / 4$ ) is ergodized and no additional laminar flux tubes with short connection length are created further inside the plasma.

Figure 4 shows the profiles of the particle flux, electron temperature and plasma potential at the target in 12/4 base mode. The profiles were measured by Langmuir probes embedded in the target tiles. In order to gain more resolution the magnetic structure was swept by $22.5^{\circ}$ in toroidal direction. This operating mode is only possible at a reduced current in the DED coils. 


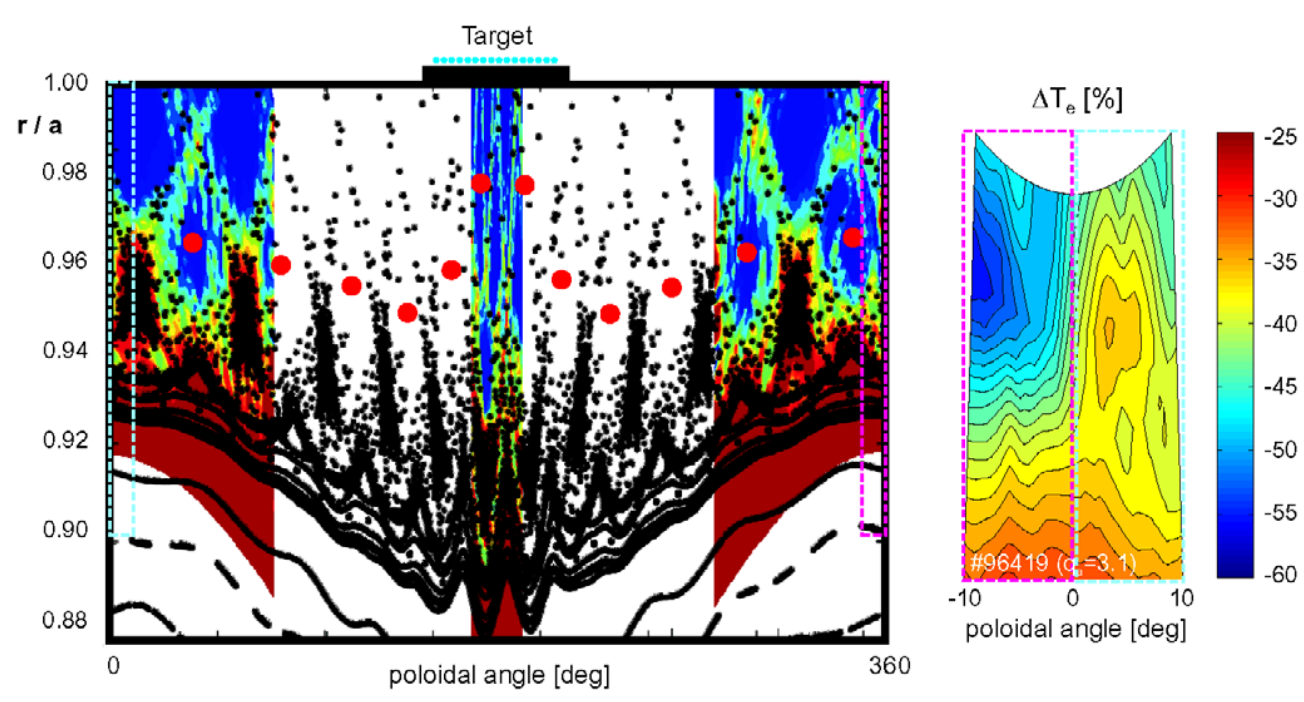

Figure 3. Laminar region in $12 / 4$ configuration $\left(q_{\mathrm{a}}=3.1\right)$. The connection length varies from one poloidal turn (blue) to five poloidal turns (red). The red dots indicate a flux tube connecting within one poloidal turn to the target plates. On the right the drop in electron temperature with DED from the ECE imaging diagnostic is shown. The dashed boxes indicate the observation volume.
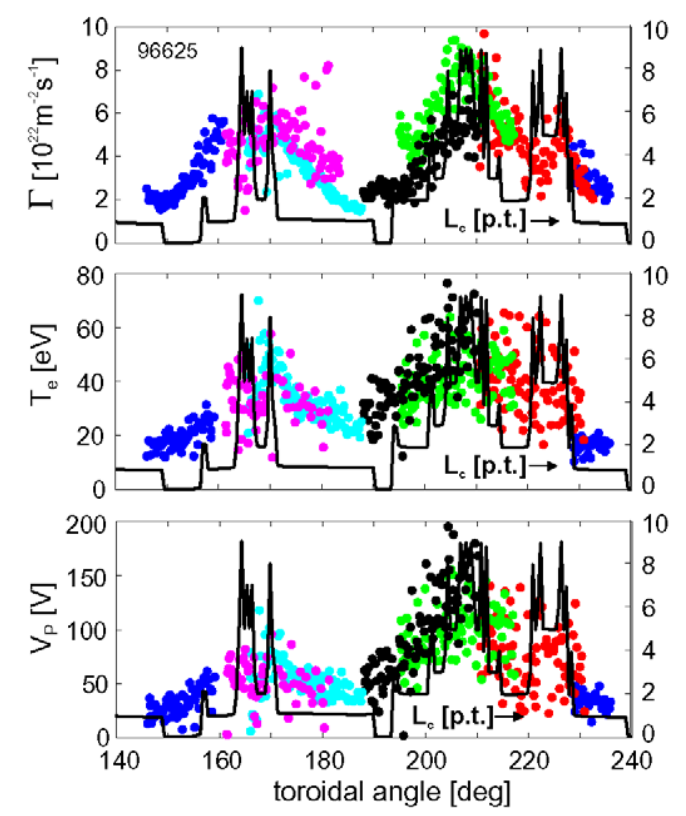

Figure 4. Toroidal profiles of particle flux $\Gamma$, electron temperature $T_{\mathrm{e}}$ and plasma potential $V_{\mathrm{P}}$ (\#96625). The colours indicate different target probes. The black curve is the connection length $L_{\mathrm{c}}$ in poloidal turns.

The profiles were taken at an edge safety factor of $q_{\mathrm{a}}=3.1$, a divertor coil current of $6.1 \mathrm{kA}$, and $P_{\mathrm{NBI}}=300 \mathrm{~kW}$. The magnetic topology at the target is shown by the connection length profile. The highest particle flux and temperature is found at the positions where the finger structure connects to the target. These field lines transport particles and heat from the vicinity 

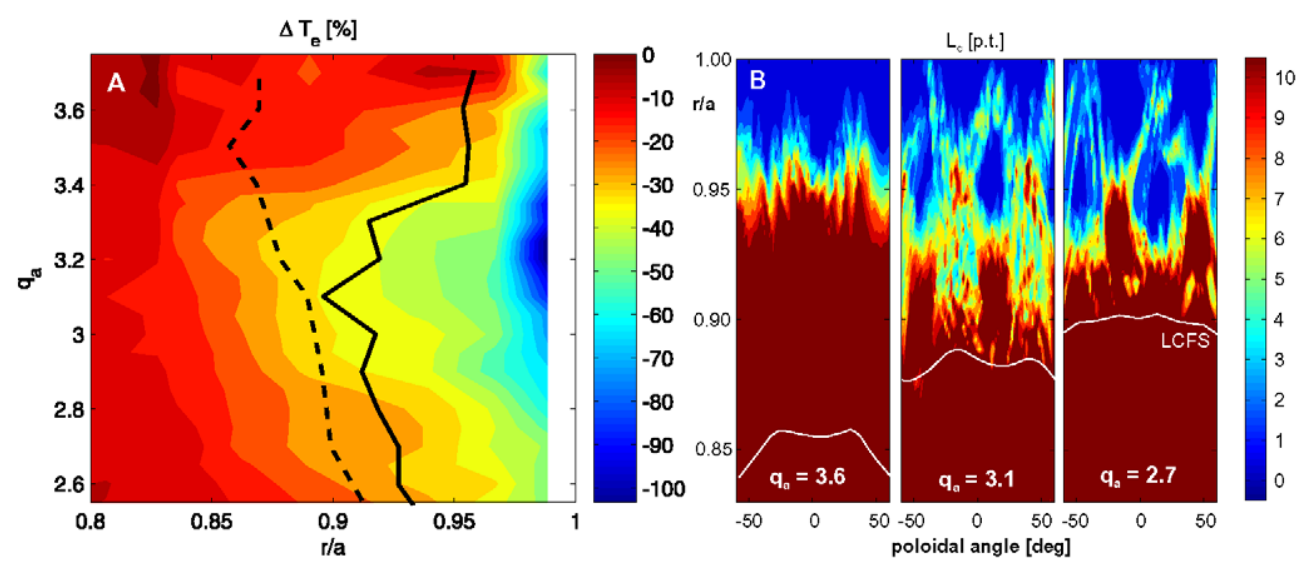

Figure 5. (a) Drop in the electron temperature at the LFS. The solid line represents the boundary to the laminar zone $\left(L_{\mathrm{c}}=5\right.$ p.t.). The dashed line is the LCFS. The region in between these two lines is the ergodic layer. $(b)$ Connection length for different safety factors. Temperatures in $(a)$ are measured at $\theta=0^{\circ}$.

of the last closed flux surface (LCFS) to the target. The adjacent flux tubes are filled by cross field diffusion and show an exponential decrease of temperature and particle flux as can be seen, for example, between $\phi=172^{\circ}$ and $\phi=188^{\circ}$. The same behaviour was found in the $3 / 1$ base mode [10]; however, the position of the probes does not allow measurement of full profiles in this case. The variation of the plasma potential with the connection length allows us to estimate the plasma potential inside the ergodic region close to the LCFS to be up to $150 \mathrm{~V}$. Taking into account the width of the perturbed volume of about $30 \mathrm{~mm}$ and a potential of about $30 \mathrm{~V}$ in the far SOL the electric field can be estimated to $4 \mathrm{kV} \mathrm{m}^{-1}$. Compared to the limiter reference case the inversion point of the plasma potential is moved further to the plasma centre (cf [14]). This might be expected since the laminar region is extended by the divertor.

\section{The role of the safety factor}

The ergodic divertor is based on a resonant mechanism. Since the DED coils cover only a part of the poloidal circumference, the perturbation spectrum is not singular. Poloidal mode numbers with a relevant contribution to the perturbation field range between $m=9$ and $m=14$ for the 12/4 configuration. The distance between the resonance layer and the DED coils defines the field structure. This distance and the number of resonance layers in the plasma can be varied by changing the safety factor. Also the distance between rational $q$ surfaces at the HFS influences the degree of ergodization. Thus an increase in the plasma pressure giving an increase in the Shafranov shift leads to further separation of the resonance layers and counteracts on the creation of an ergodic region in the plasma edge. The choice of the magnetic structure is, therefore, not independent from the plasma parameters.

Figure 5(a) shows the temperature drop during divertor operation as measured by the ECE diagnostic in the $12 / 4$ configuration. The safety factor was varied by a plasma current ramp. A radial resolution of about $10 \mathrm{~mm}$ was achieved by slight variation of the toroidal magnetic field. The black solid line indicates the boundary between the laminar and the ergodic regions ( $L_{\mathrm{c}}=5$ p.t.), the dotted line gives the position of the LCFS. The change in the laminar structure is shown in figure $5(b)$. 

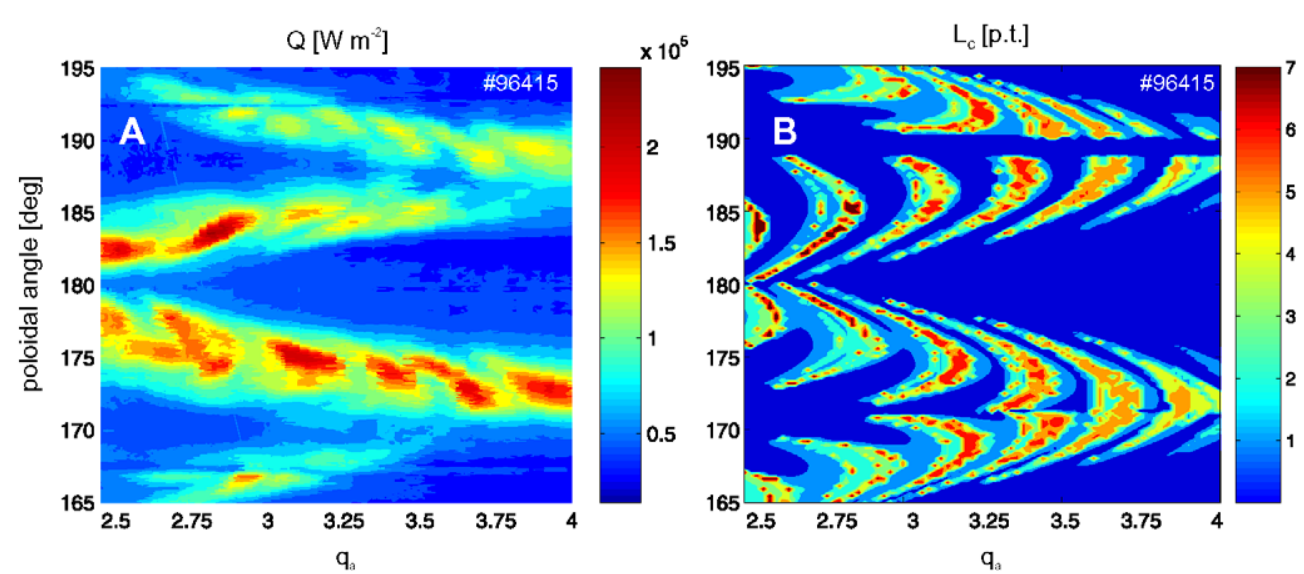

Figure 6. (a) Influence of the safety factor on the heat flux pattern (\#96415). (b) Connection length $L_{\mathrm{c}}$ on the target plates in poloidal turns.

For large safety factor most of all of the relevant resonance layers are inside the plasma. A broad ergodic region is formed, but no significant change on the radial transport is to be expected since the field line diffusion coefficient stays below $1 \times 10^{-6} \mathrm{~m}$. A temperature drop is only observed inside the laminar region.

The plasma perturbation reaches a maximum for a safety factor just above 3.0. In this case the main resonance layers are close to the perturbation coils. The temperature reduction now extends over several centimetres. However, a flattening of the temperature profile is not seen. The ergodic region is about $1 \mathrm{~cm}$ in width and a change of the gradients cannot be detected with this type of diagnostic. The field line diffusion coefficient exceeds $D_{\mathrm{FL}}=1 \times 10^{-6} \mathrm{~m}$ only within a few millimetres, such that profile changes caused by an enhanced radial transport are not expected. It is the extension of the laminar zone which causes the temperature drop. Below $q_{\mathrm{a}}=3.0$ the laminar zone starts to decrease again in size and accordingly the temperature drop is restricted further to the plasma edge. However, it is remarkable that the temperature further inside the plasma is unchanged. Consistent with this, there is no degradation of the energy or particle confinement observed, a result also reported from Tore Supra [1] and attributed to an intrinsic transport barrier.

The appearance of new resonance layers inside the plasma with increasing $q_{\mathrm{a}}$ is reflected on the heat flux pattern given in figure 6(a). At low $q_{\mathrm{a}}$ the strike lines lie close together such that they can not be distinguished, resulting in four strike lines on the target. With increasing $q_{\mathrm{a}}$ the strike lines separate and form a set of eight strike lines until they join again at high $q_{\mathrm{a}}$. The strong link between heat flux and magnetic topology is seen from figure $6(b)$ giving the connection length pattern on the target. Heat flux maxima are found where field lines from the ergodic region connect to the target (large $L_{\mathrm{c}}$ ). Besides the dependence of the coarse position of the strike lines on $q_{\mathrm{a}}$, additionally a substructure on the heat flux pattern is seen which correlates to rational values of the safety factor. This substructure is defined by the last island chain in front of the target $[16,17]$.

\section{Electric field and plasma rotation}

DED operation causes a significant spin-up of the toroidal plasma rotation [7]. This increase in rotation is independent of the rotation of the perturbation field and is also seen for DC operation. It was observed for both operating scenarios, $3 / 1$ and 12/4. 


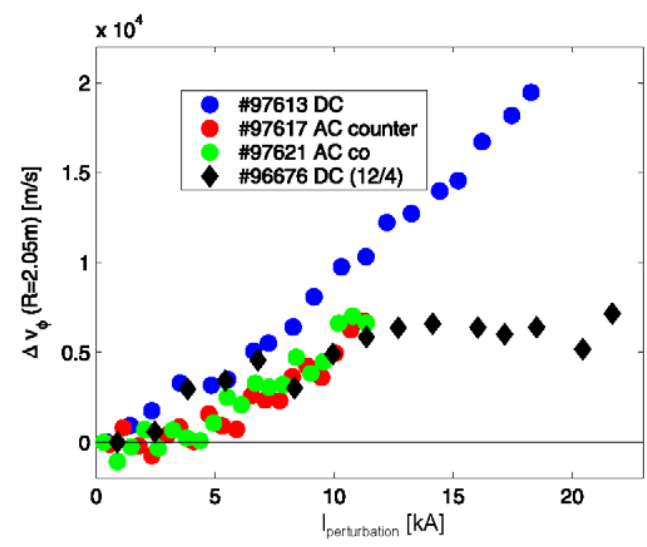

Figure 7. Increase of the toroidal rotation as a function of the perturbation amplitude.

Figure 7 shows the change of the toroidal velocity as measured by CXRS at $R=2.05 \mathrm{~m}$. This position corresponds to $r / a=0.64$ for $3 / 1$ and to $r / a=0.81$ for the $12 / 4$ base mode where the plasma was shifted towards the HFS. The abscissa gives the perturbation current which is proportional to the radial magnetic field of the divertor:

$$
I_{\text {perturbation }}=\left\{\begin{array}{l}
\frac{8}{n} \times I_{\text {coil }} \quad(\mathrm{DC}), \\
\frac{8}{n} \times \frac{2}{\pi} \times I_{\text {coil }} \quad(\mathrm{AC}) .
\end{array}\right.
$$

The perturbation current is $64 \%$ of the coil current amplitude in AC operation caused by the $90^{\circ}$ phase shift between the two feeder systems.

The gradient of the rotation profile in the measured volume (centre to $R=2.05 \mathrm{~m}$ ) does not change during the speed-up. Thus, the source for the rotation spin-up is located at the plasma edge, most likely inside the perturbed volume. Momentum transfer from the rotating perturbation field [18] can be excluded since the spin-up is independent of the rotation direction of the DED. The poloidal velocity measured from $\mathrm{C}$ III radiation at the LFS increases with the perturbation amplitude also independently from the operation mode [19] pointing towards a change of the radial electric field in the perturbed volume.

The electric field can be modified by two main changes in the plasma edge: the extension of the laminar region and the creation of an ergodic layer. Inside the laminar region the plasma potential is given by the boundary condition at the target. Thus, as in the usual SOL, it is defined as $V_{\mathrm{P}}=3 \times k T_{\mathrm{e}} / e$. However, as one can see from figure 3 , the laminar zone has a complex mixture of field lines with different connection length which makes an estimate for the electric field non-trivial. Inside the ergodic region the radial transport is faster for electrons than for ions because of the projection of the parallel transport in radial direction. The electric field is generated by the same mechanism as for the plasma-wall contact: it has to prevent electron outflow in order to satisfy the ambipolarity constraint. The current expected in an ergodic region is (cf $[20,21])$ :

$$
j_{r}=\sigma_{\mathrm{erg}}\left(-E_{r}+\frac{T_{\mathrm{e}}}{e L_{\mathrm{n}}}+\frac{1.71 T_{\mathrm{e}}}{e L_{\mathrm{T}}}\right)
$$

with $L_{\mathrm{n}}$ and $L_{\mathrm{T}}$ being the radial gradient length of the density and electron temperature, respectively. The effective conductivity $\sigma_{\text {erg }}$ is caused by the radial projection of the parallel conductivity. 
The electric field in the ergodic region and the accompanying change in toroidal rotation can be estimated by assuming the radial electron current to be compensated by the neoclassical radial current [23]. An equation for the toroidal velocity follows from the ambipolarity constraint (see e.g. equation (7) in [24]). The current (2) results in an additional source in the velocity equation. For a first estimate we reduce equation (9) in [24] and include the source term originating from equation (2):

$$
\frac{1}{r} \frac{\partial}{\partial r}\left(r \eta \frac{\partial v_{\phi}}{\partial r}\right)+\frac{B_{\chi} j_{r}}{4 \pi^{2} R a} \delta\left(r-a-\Delta_{\text {erg }}\right)=0 .
$$

The current $j_{r}$ is restricted to the ergodic region between $a+\Delta_{\text {erg }}$ and $a$. The momentum input in the plasma core is not included since we restrict our estimate to the offset in toroidal rotation $v_{\phi}$ at the plasma edge. The radial transport is described by an anomalous viscosity $\eta$. Integration of equation (3) together with $E_{r}=B_{\theta} v_{\phi}-B_{\phi} v_{\theta}+k T_{\mathrm{e}}\left(L_{\mathrm{n}}^{-1}+L_{\mathrm{T}}^{-1}\right) / e$ gives values for the toroidal rotation as well as for the electric field. Using the characteristic values for the temperature and density gradients and the neoclassical value for the poloidal velocity together with $D_{\mathrm{FL}}=2 \times 10^{-6} \mathrm{~m}$ we get $E_{r} \approx 3 \mathrm{kV} \mathrm{m}^{-1}$ and $v_{\phi}=2 \times 10^{4} \mathrm{~m} \mathrm{~s}^{-1}$.

Independent of the exact mechanism for the modification of the edge electric field, the perturbation by the DED leads to a shift of the inversion point of the electric field towards the plasma centre. A fact also known, for example, from measurements at TEXT [14]. The shift of the inversion point during DED operation is indicated by a change in the probe floating potential at the LFS [22]. As already shown in section 2, the plasma 'scrape-off layer' is extended by up to several centimetres. The electric field in this region can be derived from the plasma potential at the target plates together with the knowledge of the magnetic field structure and is of the order of a few kilovolts per metre. Systematic measurements of the electric field inside the ergodic region will be possible after the installation of a fast reciprocating probe in summer 2005.

Indications were found that the change of the plasma rotation also affects the energy confinement. Discharges with strong counter-NBI exhibit an increase of about $10 \%$ in the energy confinement time at $50 \%$ of the perturbation amplitude. Discharges with slow DED current ramps show that the increase of $\tau_{E}$ is proportional to the perturbation amplitude (as is the change in toroidal rotation) indicating a relation to an increased velocity shear at the plasma edge.

\section{Particle recycling}

The particle recycling is strongly affected by the perturbation field as can be seen, for example, from the $\mathrm{C}_{\text {III }}$ radiation shown in section 2 . This has consequences on the density regimes and the density limit as well as on the impurity generation and transport properties.

\subsection{Density limit}

Investigations on density regimes and density limit were done in the $3 / 1$ base mode. As stated before, the magnetic structure depends on the plasma pressure and does, therefore, change during density ramps. The identification of different divertor regimes is therefore challenging. The 3/1 configuration was chosen for this type of analysis because the coarser magnetic structure is more 'resistant' to changes in the Shafranov shift than the finer structures in the $12 / 4$ configuration. The divertor density in $3 / 1$ configuration shows the existence of three regimes, like it is observed in the poloidal divertor: low recycling with linear dependence of 

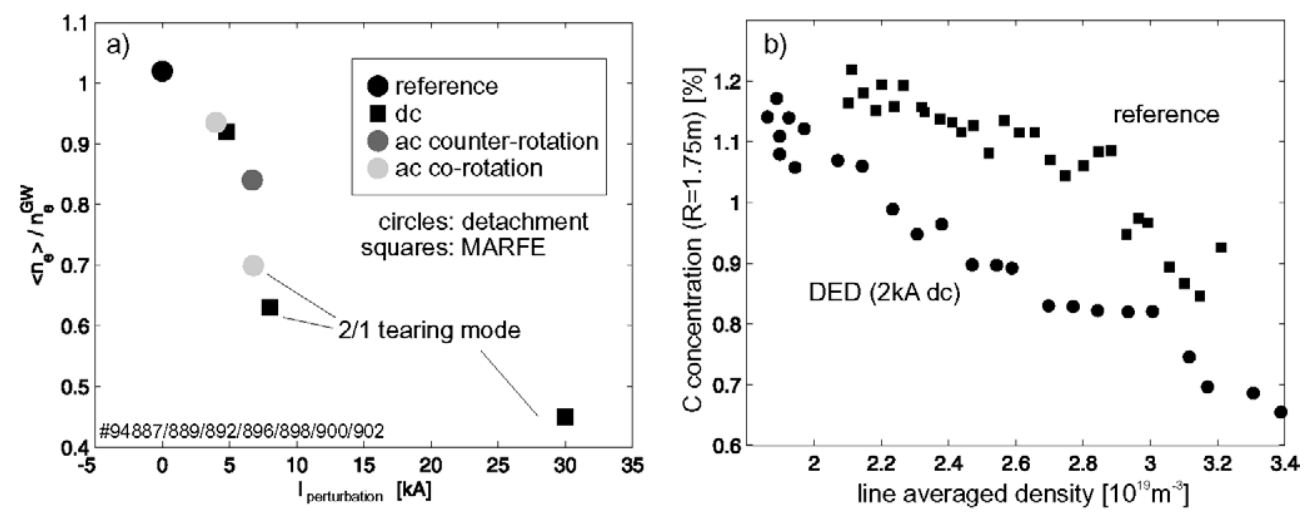

Figure 8. (a) Density limit as a function of the perturbation amplitude in ohmic plasmas. (b) Carbon concentration for the counter-NBI scenario in $3 / 1$ base mode with $I_{\text {perturbation }}=16 \mathrm{kA}$ (coil current $2 \mathrm{kA}$ ). Discharge parameter were $I_{\mathrm{P}}=300 \mathrm{kA}, B_{\mathrm{t}}=2.25 \mathrm{~T}, P_{\mathrm{tot}}=2.0 \mathrm{MW}$ (reference: \#97957, DED: \#97958)

the target density on the line averaged density, high recycling with a strong increase of the target density and finally the detachment phase [10]. Interestingly, detachment sets in at temperatures around $10 \mathrm{eV}$. Such a detachment without volume recombination was also observed in Tore Supra [25] and in the island divertor of W7-AS [26] and might be related to frictional forces between the flowing plasma streams in neighbouring divertor legs.

During limiter operation the recycling takes place at the toroidal belt limiter positioned $45^{\circ}$ below the equatorial plane at the low field side. The density limit in neutral beam heated limiter discharges is caused by the excitation of a MARFE. This type of density limit is strongly affected by the particle recycling at the HFS target plates [27]. Plasma detachment in limiter discharges is only observed in ohmic or marginally heated discharges. The DED concentrates the recycling to the HFS and thus establishes an additional heat sink. Figure 8(a) shows the density limit in terms of the Greenwald fraction as a function of the perturbation current for cases with DC and AC perturbation fields (3/1 base mode) for ohmic discharges. The density limit is reduced for all discharges with DED operation, with a stronger reduction in the presence of a $2 / 1$ tearing mode. Remarkably, in the DC cases the limit is given by the appearance of a MARFE, whereas for the AC cases $\left(f_{\mathrm{DED}}= \pm 1.0 \mathrm{kHz}\right)$ the particle recycling is more uniform and detachment sets in at the density limit. In neutral beam heated discharges, even an increase of the MARFE limit with DED operation has been observed [28].

\subsection{Impurity screening}

A significant reduction of the carbon concentration in the plasma core is seen in the $3 / 1$ configuration. Figure $8(b)$ shows the carbon concentration measured by CXRS at the plasma centre as a function of the line averaged density in a $3 / 1$ base mode discharge with dominant counter NBI $\left(P_{\mathrm{NBI}}(\right.$ counter $)=1.3 \mathrm{MW}$ and $\left.P_{\mathrm{NBI}}(\mathrm{co})=0.4 \mathrm{MW}\right)$. During DED operation $\left(I_{\text {perturbation }}=16 \mathrm{kA}\right)$ a reduction of the carbon concentration by up to $30 \%$ is achieved. This is still a small effect compared to the strong reduction of carbon reported from Tore Supra by about a factor of 5 at maximum divertor current [15]. However, the decontamination is a strong function of the perturbation level and for a current of $15 \mathrm{kA}$ in the Tore Supra ED a similar decontamination efficiency as observed with the DED is reported [15]. A reduction of the impurity contamination in the $12 / 4$ configuration was not observed. 
Two competing mechanisms influence the impurity contamination in the plasma core: the source strength and the screening capability of the plasma edge region. Compared to the limiter reference case a reduction of the source strength during DED operation is not self-evident. Although the temperature in the laminar region is reduced, the particle flux and temperature at the strike lines are comparable to those in a limiter discharge. This picture might change in the high recycling regime. A detailed analysis on this issue should be performed by employing a multi-fluid code including neutral particle transport in three-dimensional geometry like EMC3/Eirene [29].

In a simple approach the screening capability of the edge plasma can be quantified by comparing the ionization length of the neutrals $\lambda_{i}$ with the width of the screening layer $\lambda_{s}$. The definition of the latter is not easy because of the non-uniform magnetic structure (figures $1(b)$ and $2(b)$ ). For $m / n=12 / 4$ the particles mainly recycle into the finger structures and therefore into field lines, which come close to the LCFS. On the other hand, in the $3 / 1$ base mode the emission region for $\mathrm{C}$ III and $\mathrm{H}_{\alpha}$ is the laminar layer with short connection to the target. Defining a local screening width $\lambda_{\mathrm{s}}$ equal to the width of the laminar zone in the emission regions shows that $\lambda_{\mathrm{s}}$ (limiter) $\approx \lambda_{\mathrm{s}}(12 / 4)<\lambda_{\mathrm{s}}(3 / 1)$. This analysis takes only the screening capability of the laminar region into account, but the ergodic region might also contribute to impurity screening as shown in [30].

\section{Conclusion and outlook}

From the divertor point of view we can conclude that the 12/4 configuration has the beneficial property to restrict the perturbed volume to the plasma edge. However, this configuration suffers from a low ergodization caused by the large separation of the resonance layers on the HFS. This separation is even increased in strongly heated discharges with high poloidal beta. Enhancement of the radial transport could only be seen poloidally localized. In contrast, the $3 / 1$ configuration has a large laminar region and therefore a large divertor volume. But, the excitation of tearing modes at already low perturbation amplitude in this configuration influences the plasma edge. Recently, it was found that the threshold can be increased in special heating scenarios which make this configuration also accessible for divertor studies, although less flexible. The $6 / 2$ base mode will merge the beneficial properties of the two extreme configuration. This coil set-up will go into operation by the end of 2005 .

The DED can contribute to the physics in divertor configurations where stochastic field structures play a role, namely the helical and the island divertor in stellarators. Similarities, such as the detachment behaviour, are already found and impurity transport is also an important issue. A detailed analysis of the transport properties in ergodic layers does not only contribute to the evaluation of divertor concepts but also helps in the understanding of the mechanisms leading, for example, to ELM suppression or fast heat transport during disruptions.

\section{References}

[1] Ghendrih Ph et al 2002 Nucl. Fusion 421221

[2] Masuzaki S et al 2002 Nucl. Fusion $\mathbf{4 2} 750$

[3] König R et al 2002 Plasma Phys. Control. Fusion 442365

[4] Evans T E et al 2004 Phys. Rev. Lett. 92235003

[5] Wolf R C et al 2005 Nucl. Fusion at press

[6] Finken K H et al 2004 Plasma Phys. Control. Fusion 46 B143

[7] Finken K H et al 2005 Phys. Rev. Lett. 94015003

[8] Westerhof E et al 2005 32nd EPS Conf. on Plasma Physics vol 29C (ECA) P-4.071

[9] 1997 Dynamic ergodic divertor Fusion Eng. Des. 37335 (Special issue) 
[10] Lehnen M et al 2005 J. Nucl. Mater. 337-339 171

[11] Unterberg B et al 2004 The impact of the dynamic ergodic divertor on plasma edge structure and transport in the tokamak TEXTOR 20th IAEA Conf. on Fusion Energy EX/P5-33

[12] Abdullaev S S et al 1999 Phys. Plasmas 6153

[13] Schmitz O et al 2005 32nd EPS Conf. on Plasma Physics vol 29C (ECA) P-1.022

[14] Yang X Z et al 1991 Phys. Fluids B 33448

[15] Ghendrih Ph, Grosman A and Capes H 1996 Plasma Phys. Control. Fusion 381653

[16] Wingen A et al 2005 Plasma Phys. Control. Fusion submitted

AQ4 [17] Jakubowski M et al 2005 32nd EPS Conf. on Plasma Physics vol 29C (ECA) P-1.021

[18] Finken K H et al 2001 Nucl. Fusion 41503

[19] Busch C et al 2005 32nd EPS Conf. on Plasma Physics vol 29C (ECA) P-1.016

[20] Harvey R W et al 1981 Phys. Rev. Lett. 47102

[21] Kaganovich I and Rozhansky V 1998 Phys. Plasmas 53901

[22] Jachmich S et al 2005 32nd EPS Conf. on Plasma Physics vol 29C (ECA) P-2.033

[23] Rogister A 1994 Phys. Plasmas 1619

[24] Nicolai A et al 2004 Nucl. Fusion 44 S93

[25] Gunn J P et al 1999 Plasma Phys. Control. Fusion 41 B243

[26] Wenzel U et al 2004 Nucl. Fusion 441130

[27] de Vries P et al 1998 Phys. Rev. Lett. 803519

[28] Liang Y et al 2005 Phys. Rev. Lett. 94105003

[29] Kobayashi M et al 2004 Nucl. Fusion 44 S64

[30] Tokar M et al 1997 Plasma Phys. Control. Fusion 39569 


\section{QUERIES}

Page 2

AQ1

Please be aware that the colour figures in this article will only appear in colour in the web version. If you require colour in the printed journal and have not previously arranged it, please contact the Production Editor now.

Page 11

AQ2

Please provide volume number and page range for ref [5].

Page 11

AQ3

Please provide names of the author, if any.

\section{Page 12}

AQ4

Please provide volume number and page range for ref [16]. 